\title{
Behavioral State Control through Differential Serotonergic Inhibition in the Mesopontine Cholinergic Nuclei: A Simultaneous Unit Recording and Microdialysis Study
}

\author{
Mahesh M. Thakkar, Robert E. Strecker, and Robert W. McCarley \\ Department of Psychiatry, Harvard Medical School, Brockton Veterans Administration Medical Center, \\ Brockton, Massachusetts 02401
}

Cholinergic neurons of the mesopontine nuclei are strongly implicated in behavioral state regulation. One population of neurons in the cholinergic zone of the laterodorsal tegmentum and the pedunculopontine nuclei, referred to as rapid eye movement (REM)-on neurons, shows preferential discharge activity during REM sleep, and extensive data indicate a key role in production of this state. Another neuronal group present in the same cholinergic zone of the laterodorsal tegmentum and the pedunculopontine nuclei, referred to as Wake/REM-on neurons, shows preferential discharge activity during both wakefulness and REM sleep and is implicated in the production of electroencephalographic activation in both of these states. To test the hypothesis of differential serotonergic inhibition as an explanation of the different state-related discharge activity, we developed a novel methodology that enabled, in freely behaving animals, simultaneous unit recording and local perfusion of neuropharmacological agents using a microdialysis probe adjacent to the recording electrodes. Discharge activity of REM-on neurons was almost completely suppressed by local microdialysis perfusion of the selective $5-\mathrm{HT}_{1 \mathrm{~A}}$ agonist 8-hydroxy-2-(di-n-propylamino) tetralin (8-OH-DPAT), although this agonist had minimal or no effect on the Wake/REM-on neurons. We conclude that selective serotonergic inhibition is a basis of differential state regulation in the mesopontine cholinergic nuclei, and that the novel methodology combining neurophysiological and neuropharmacological information from the freely behaving animal shows great promise for further insight into the neural basis of behavioral control.

Key words: REM sleep; serotonin; laterodorsal tegmental nucleus; pedunculopontine tegmental nucleus; mesopontine cholinergic neurons; microdialysis; single-unit recording
Several lines of evidence indicate that the activity of cholinergic neurons in the mesopontine tegmentum is involved in the generation of rapid eye movement (REM) sleep via their projections to the pontine reticular formation (PRF) (for review, see McCarley et al., 1995) and thalamus (Steriade et al., 1990). These cholinergic neurons are located in the laterodorsal tegmental and pedunculopontine tegmental nuclei (LDT/PPT) and have descending projections to sites in the PRF (Mitani et al., 1988) in which local injections of cholinergic agonists produce a REM-like state that very closely mimics natural REM sleep (George et al., 1964; Amatruda et al., 1975). Furthermore, in vitro application of low concentrations of cholinergic agonists to PRF neurons produces the same enhanced excitability and depolarization (Greene et al., 1989) seen in intracellular in vivo studies during natural REM sleep (Ito and McCarley, 1984).

As early as the 1960 s, studies transecting the neural axis localized the neural circuitry required for the generation of REM sleep to the area containing the LDT/PPT (Jouvet, 1962). More recently, local excitotoxin-induced cell body lesions of the LDT/ PPT were found to produce a decrease in REM sleep (Webster and Jones, 1988; Thakkar et al., 1995), whereas electrical stimu-

Received March 11, 1998; revised April 17, 1998; accepted April 23, 1998.

This work was supported by the Department of Veterans Affairs and by National Institutes of Health Grant MH39683. We thank Michael Gray and John Franco of the Veterans Administration Medical Center Animal Facility for providing care for the animals and Dr. P. J. Shiromani for advice on histology.

Correspondence should be addressed to Robert W. McCarley, Harvard Medical School, Brockton VAMC, 116-A, 940 Belmont Street, Brockton MA, 02401.

Copyright (C) 1998 Society for Neuroscience $\quad 0270-6474 / 98 / 185490-08 \$ 05.00 / 0$ lation of the LDT increased REM sleep (Thakkar et al., 1996). Neurochemical studies have found higher levels of pontine acetylcholine during REM sleep (Kodama et al., 1992; Leonard and Lydic, 1997), as well as changes in pontine acetylcholinesterase after REM deprivation (Thakkar and Mallick, 1991). Electrophysiological studies reveal that a subpopulation of LDT/PPT neurons preferentially discharges just before and during REM sleep (El Mansari et al., 1989; Steriade et al., 1990; Kayama et al., 1992). The LDT/PPT neurons that fire preferentially during REM sleep are often termed REM-on neurons. A separate subpopulation of LDT/PPT cholinergic neurons is active during both wakefulness and REM sleep and is referred to as Wake/REM-on neurons.

With respect to control of mesopontine cholinergic activity, monoaminergic neurons from the nearby noradrenergic locus ceruleus and serotonergic dorsal raphe nucleus (DRN) exhibit a pattern of discharge activity that is nearly opposite to that of the cholinergic LDT/PPT neurons; discharge is greatest during waking, declines during non-REM sleep, and virtually ceases before and during REM sleep for both DRN (McGinty and Harper, 1976; Lydic et al., 1987; Jacobs and Fornal, 1991) and locus ceruleus (Hobson et al., 1975; Foote et al., 1983). This inverse correlation with REM sleep led to suggestions that noradrenergic (McCarley and Hobson, 1975) and serotonergic activity (McGinty and Harper, 1976) might suppress REM sleep, and it formed the basis of a structural and mathematical model of REM sleep control termed the reciprocal interaction model (McCarley and Hobson, 1975). As evidence accumulated that some mesopontine cholinergic neurons were REM-promoting and REM- 
on, McCarley and colleagues suggested that it was on cholinergic neurons that monoaminergic inhibition acted during wakefulness and, through disinhibition, promoted REM-on activity (revised reciprocal interaction model, McCarley and Massaquoi, 1992; McCarley et al., 1995). It was further postulated that whereas monoamines might inhibit REM-on cholinergic neurons, Wake/ REM-on neurons might not be inhibited, thus explaining their continued activity in waking (McCarley et al., 1995). Because serotonergic activity is highest during wakefulness, the observed high discharge rate of Wake/REM-on neurons during wakefulness would not be consistent with a high level of serotonergic inhibition.

There were anatomical, physiological, and pharmacological data consistent with these hypotheses about monoaminergic actions on cholinergic neurons in the control of behavioral state, for both noradrenaline (Williams and Reiner, 1993; for review, see McCarley et al., 1995) and serotonin. Serotonergic neurons project to LDT/PPT (Semba and Fibiger, 1992; Honda and Semba, 1994; Steininger et al., 1997), and in vitro studies of identified mesopontine cholinergic neurons revealed that a subset is inhibited by $5-\mathrm{HT}$ acting at $5-\mathrm{HT}_{1 \mathrm{~A}}$ receptors (Muhlethaler et al., 1990; Luebke et al., 1992; Leonard and Llinas, 1994). Portas and McCarley (1994) showed that spontaneous extracellular levels of serotonin in the DRN during sleep and wakefulness paralleled the changes in spontaneous serotonergic neuronal discharge. Furthermore, inhibition of DRN serotonergic activity by the specific $5-\mathrm{HT}_{1 \mathrm{~A}}$ agonist 8-hydroxy-2-(di- $n$-propylamino) tetralin (8-OH-DPAT) increased REM sleep (Portas et al., 1996). Finally, microinjection of 8-OH-DPAT into the LDT or PPT decreased REM sleep (Horner et al., 1997; Sanford et al., 1994).

However, because this evidence was indirect and circumstantial, a direct test of serotonergic inhibition of LDT/PPT neurons behaviorally identified as REM-on and Wake/REM-on was needed. The present study was designed to provide this direct test using a novel methodology allowing both extracellular single-cell recording and local perfusion of neuropharmacological agents via an adjacent microdialysis probe in naturally sleeping cats. A preliminary version of this work has been presented in abstract form (Thakkar et al., 1997).

\section{MATERIALS AND METHODS}

Preparation of the chronic animals. Six male adult cats were implanted with electrodes for recording EEG, EMG, electro-oculogram (EOG), and ponto-geniculo-occipital (PGO) waves for determination of behavioral state and with two devices developed by us for simultaneous unit recording and microdialysis. Each device consisted of two 17 ga stainless steel guide cannulas and a screw-driven microdrive with two advanceable 19 ga stainless steel cannulas within the guide tubes. Within one of the advanceable cannulas was a microwire unit recording a bundle of seven $32 \mu \mathrm{m}$ and seven $64 \mu \mathrm{m}$ insulated wires, and within the other cannula was an obturator that was removed when the microdialysis probe was inserted. Dimensions of the steel guide cannulas were $1.5 \mathrm{~mm}$ outer diameter (o.d.) and $1.2 \mathrm{~mm}$ inner diameter (i.d.). The advanceable stainless steel tubes had centers with $0.7 \mathrm{~mm}$ lateral separation and dimensions of $1.1 \mathrm{~mm}$ o.d. and $0.8 \mathrm{~mm}$ i.d.

The guide tubes of one device were stereotaxically targeted above the LDT using a vertical approach with tentorectomy, and the guide tubes of the other device were targeted above the PPT using a posterior angled $\left(45^{\circ}\right)$ approach. The centers of the stereotaxic target coordinates for LDT were anteroposterior (AP), posterior 1 (P1); mediolateral (ML), 0.5 $\mathrm{mm}$; and for PPT were, AP, P1; ML, $3 \mathrm{~mm}$ (Berman, 1968). The vertical approach optimized the LDT volume available for recording, although it required removal of the tentorium, whereas the $45^{\circ}$ angled PPT approach optimized PPT access by placing electrode and microdialysis tracks at the same angle as the dorsoventral course of the PPT (parallel to the brachium conjunctivum). The two devices were fixed onto the skull with dental acrylic; the tips of the guide tubes were $5 \mathrm{~mm}$ above the target, with the microwires (California Fine Wire Co., Grove City, CA) inside the inner stainless steel tubing placed $1 \mathrm{~mm}$ above the target.

The microdialysis probes were custom-made CMA 10 probes (CMA/ Microdialysis, Acton, MA) with a polycarbonate membrane $(20,000 \mathrm{Da}$ molecular weight cutoff), a $500 \mu \mathrm{m}$ o.d., a $2 \mathrm{~mm}$ microdialysis membrane length, and a $55 \mathrm{~mm}$ shaft length. The flow rate of artificial CSF (ACSF; in mM: $\mathrm{NaCl} 147, \mathrm{KCl} 3, \mathrm{CaCl}_{2} 1.2$, and $\mathrm{MgCl}_{2} 1.0, \mathrm{pH}, 6.6$ ) through the probe was $1.5 \mu \mathrm{l} / \mathrm{min}$, which is the same flow rate used for drug perfusion. The microdialysis tubing connecting the perfusion pump with the microdialysis probe had a low and known dead space volume $(1.2 \mu \mathrm{l} /$ $10 \mathrm{~cm}$ ) (FEP-tubing, CMA/Microdialysis), so that the time of arrival of a perfused drug at the brain could be correlated with the electrographically defined sleep-wake states and unit activity of the animal. The $5-\mathrm{HT}_{1 \mathrm{~A}}$ receptor agonist $8-\mathrm{OH}-\mathrm{DPAT}$ was selected because it is the most specific agonist for this receptor, and the $10 \mu \mathrm{M}$ concentration of $8-\mathrm{OH}-$ DPAT was chosen on the basis of previous data, which also suggest a 10 -fold reduction in concentration outside the microdialysis membrane compared with the perfusion concentration (Portas et al., 1996). Although not evaluated systematically, the effects of 8-OH-DPAT reported here were reversible over time in all of the cells followed sufficiently long after infusion.

Simultaneous extracellular single-unit recording and perfusion. After postoperative recovery, each animal was habituated to the recording chamber for at least 1 week. Then, extracellular unit recording was begun using standard extracellular unit recording techniques. A recording system (MDA-4; BAK Electronics, Germantown, MD) included a highinput impedance head stage preamplifier, an amplifier, high- and lowpass filtering (500-5000 $\mathrm{Hz}$ bandpass used), and a window discriminator (DDIS-1; BAK Electronics). The amplified unit signal, the discriminator window, and the window pulse were monitored on a storage oscilloscope. The electrographic signals and the window pulse were displayed on an inkwriter, and they, together with the unit signal, were digitally tape recorded [Vetter 3000A seven-channel digital recording adapter (88.2 $\mathrm{KHz}$ ) with a Sony (Tokyo, Japan) videocassette recorder]. The untransformed unit activity and window discriminator pulse were also fed into a 16-channel analog-to-digital board $(150 \mathrm{KHz}$; Datawave Technology) on a Pentium microcomputer for further analysis using Experimental Workbench 5.01 software (Datawave Technology). The firing rates were calculated using at least six $10 \mathrm{sec}$ epochs for each of the different behavioral states.

After encountering a unit, the microdialysis probe was then inserted, ACSF perfusion was begun, and after allowing at least $12 \mathrm{hr}$ postinsertion recovery, the experiment was begun. The microdrive was slowly advanced (25-50 $\mu \mathrm{m}$ steps) until an REM-on or Wake/REM-on neuron was recorded over one complete wake-non-REM-REM sleep cycle. Perfusion of $10 \mu \mathrm{M}$ 8-OH-DPAT was then started and continued for 30-45 min. Throughout these procedures, the animal moved freely without restraint, and a unit signal/noise ratio $>2: 1$ was maintained. EEG, EOG, EMG, PGO, and the window-discriminated single neuronal activity were recorded simultaneously on five different channels of the polygraph, and samples of the untransformed and window-discriminated unit activity and electrographic records were recorded on a digital tape recorder. The purpose of the present study was to use relatively brief perfusions that would allow estimation of the effect on single units in the vicinity of the probe and to not attempt longer-duration perfusions that would affect large populations of neurons and hence modify behavioral state. Subsequent preliminary experiments (Strecker et at., 1998) showed that longer-duration 8-OH-DPAT perfusions did reduce REM sleep.

Histology and identification of the recording zone. After completion of the recordings, the recording site was marked with a small electrolytic lesion produced by passing DC (100 $\mu \mathrm{A}$ for $20 \mathrm{sec})$ through the wire from which the maximum number of units was recorded. One week later, the animals were deeply anesthetized with sodium pentobarbital and perfused with $500 \mathrm{ml}$ of saline followed by $4 \%$ formaldehyde in $0.1 \mathrm{M}$ PBS. The brainstem was isolated, blocked, and placed overnight in the $4 \%$ formaldehyde-PBS solution. It was then transferred to $20 \%$ sucrose- $0.1 \mathrm{M}$ PBS for at least $4 \mathrm{~d}$. Forty micrometer sagittal sections were cut on a freezing microtome. A series of one-in-three sections were subsequently stained for the cholinergic marker NADPH and counterstained with neutral red, as described previously (Luebke et al., 1992). The sagittal section with the lesion site tip was used for reconstruction of the recording zone. Identification of recorded neurons as being cholinergic or noncholinergic is tentative. It should be acknowledged that the only way to be completely certain that cells with a specific state-related discharge 

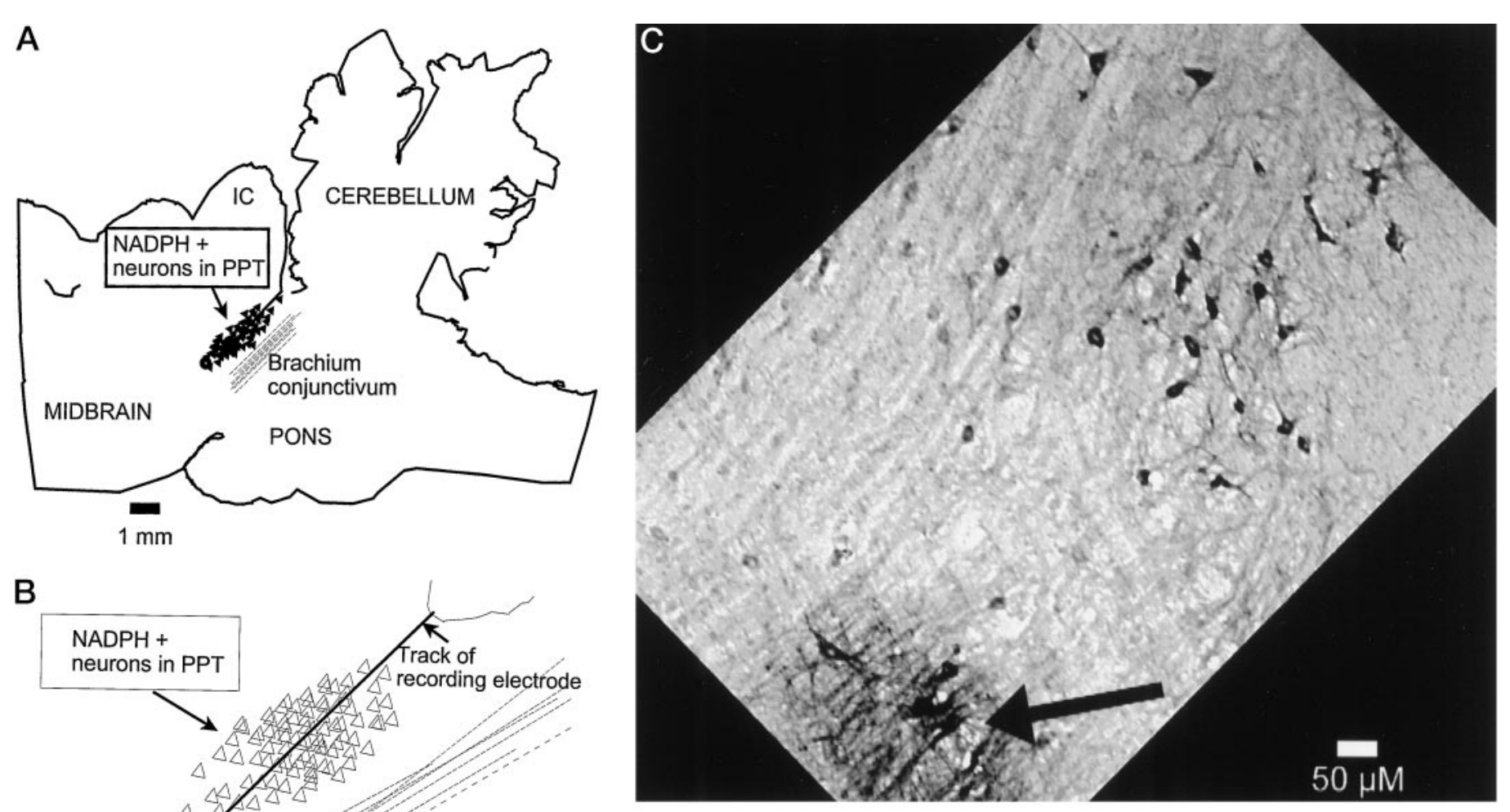

B

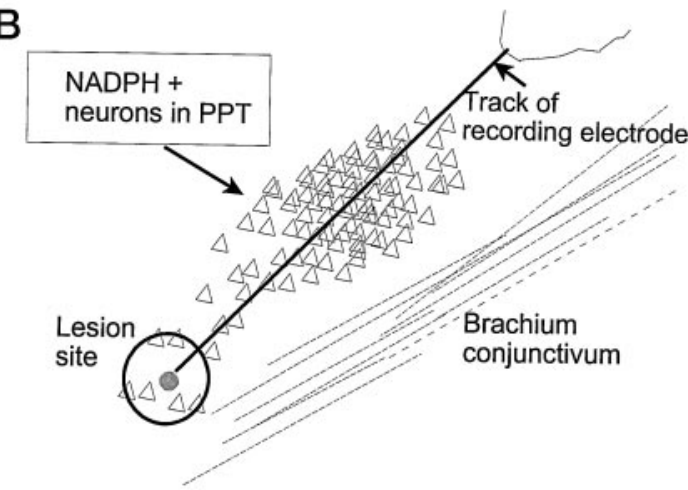

Figure 1. A, Low-power camera lucida tracing from a sagittal section showing an electrode track and lesion site in PPT localized in a zone of neurons ( filled triangles) staining positively for NADPH-diaphorase $(\mathrm{NADPH}+$ ), a cholinergic marker (Luebke et al., 1992). B, Higher-power camera lucida drawing of recording track and diaphorase-positive neurons whose soma (open triangles) were in the plane of this section or in that of the two NADPH diaphorase-stained sections to either side. $C$, Photomicrograph of track and lesion site (same angle as in $A, B$ ) and NADPH+ neurons (dark somata). $I C$, Inferior colliculus.

pattern are cholinergic would be to use intracellular recording in restrained animals combined with intracellular labeling of the recorded cell for later ChAT immunohistochemistry, which thus far is a technique not used successfully by any worker in the field and one not compatible with the goals of the present study, i.e., using 8-OH-DPAT perfusion in freely moving animals. Other criteria for identification of cholinergic neurons are discussed in Results in conjunction with presentation of data.

Analysis of the discharge activity across behavioral states. The discharge rates of the neurons were classified in five different states (Ursin and Sterman, 1981): (1) active awake (AW); (2) quiet awake (QW); (3) non-REM sleep; (4) REM sleep without eye movements (REM-); and (5) REM sleep with eye movements (REM+). AW was defined by the presence of an activated (desynchronized) EEG with somatic movement (reflected by EMG artifact) and marked eye movements. QW was the state with an activated (desynchronized) EEG and an EMG tonic activity but no somatic movement and few or no eye movements. The onset of non-REM sleep was defined by EEG synchronization without any transient periods of desynchronization. The onset of REM sleep was defined by the concomitant presence of EEG desynchronization, the appearance of the first eye movement, muscle atonia, and PGO activity. REM - was analyzed separately from REM +. Arousal from the REM sleep state was indicated by the reappearance of muscle tone and cessation of PGO activity. Each cell was recorded for at least one complete cycle of wake-non-REM sleep-REM-wake. The firing rates were calculated for different behavioral states using at least six epochs of $10 \mathrm{sec}$ duration for each state. REM-on neurons were defined as those with REM+ discharge rates twice or more than that of both non-REM and active wakefulness. Wake/REM-on neurons were defined as those with discharge rates in both REM+ and AW states greater than in non-REM but with REM+/active wakefulness discharge ratios $<2$.

\section{RESULTS}

\section{Anatomical localization}

Figure 1 shows the histological localization of a lesion from the microwire bundle (the dialysis probe tip was visible in a slightly more lateral section) and the descent track in PPT. Note that the descent courses through the dorsoventral plane of cholinergic neurons running parallel to the brachium conjunctivum. Thirtyfour neurons were recorded across all behavioral states and during ACSF and 8-OH-DPAT perfusion. Of the 34 neurons recorded in mesopontine cholinergic zones, nine (26\%) were REM-on neurons (Figs. 2, 3) and 25 (74\%) were Wake/REM-on neurons (Fig. 4). Eleven neurons and the companion microdialysis probes were histologically localized to the LDT, and 22 neurons and companion probes were localized to the PPT. The LDT had a slightly higher percentage of recorded REM-on neurons $(n=4 ; 36.4 \%)$ than did the PPT $(n=5 ; 21.7 \%)$. Conversely, a slightly higher percentage of Wake/REM-on neurons were in PPT $(n=18 ; 78.3 \%)$ than in LDT $(n=7 ; 63.6 \%)$, but these differences were not statistically significant (Fisher's exact test).

\section{Discharge activity}

Seven of the nine (78\%) REM-on units had relatively longduration action potentials $(\sim 1 \mathrm{msec})$, whereas only two $(22 \%)$ had shorter-duration action potentials $(\sim 0.5 \mathrm{msec})$ (Fig. 2$)$. The 

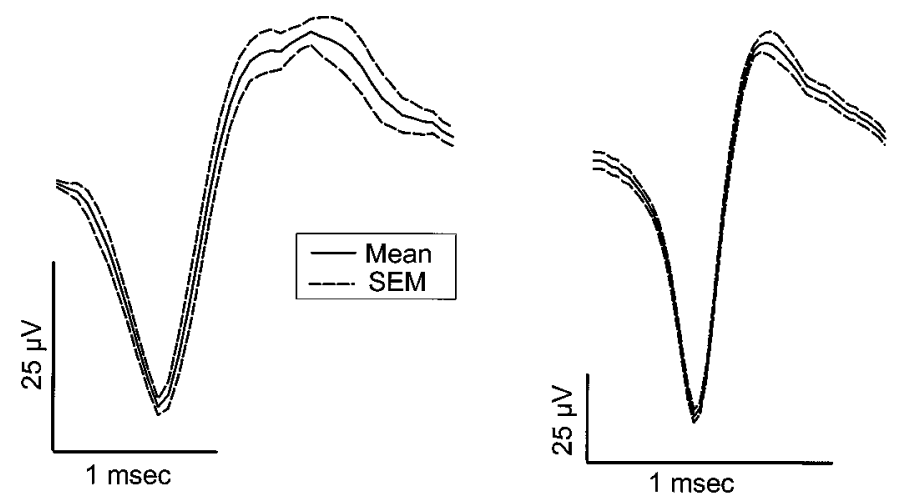

Figure 2. Average \pm SEM of 10 digitized wave forms from each of two representative REM-on units. In the REM-on population most units ( 7 of 9) showed a long-duration action potential like that shown in the left contrasted with a short-duration action potential (2 of 9) shown in the right. Note different time axes and voltage calibrations (noise background was $\sim 10 \mu \mathrm{V}$ for each unit).

duration range was $0.5-1.2 \mathrm{msec}$, and the median was $1.0 \mathrm{msec}$ (action potential only, not after hyperpolarization). The proportion of neurons with long- and short-duration action potentials was approximately the same in Wake/REM-on neurons [19 of 25 $(76 \%)$ had long-duration action potential]. Figure 3 shows the inkwriter record of the discharge activity of a sample REM-on neuron in the freely behaving animal during active wakefulness, non-REM, and REM sleep. Figure 3 further shows that for this same neuron and same REM period, the addition of 8-OH-DPAT to the perfusate almost completely suppressed the activity of this neuron. Figure 4 shows the state-related activity of a Wake/ REM-on neuron and the contrasting absence of suppressive effects of 8-OH-DPAT.

Table 1 summarizes the discharge rate statistics for the neurons recorded. For REM-on neurons, rates in REM+ were statistically different (using the Mann-Whitney $U$ test) from AW ( $p=0.027)$, QW $(p=0.005)$, and non-REM sleep $(p=0.009)$. For Wake/ REM-on neurons, rates in REM + and AW were significantly higher than in non-REM sleep $(p=0.002$ and $p=0.004$, respectively), whereas rates in AW did not differ with the rates in $\mathrm{REM}+(p=0.76)$.

\section{Differential effects of 8-OH-DPAT perfusion}

The differential effects of 8-OH-DPAT perfusion were striking. Table 1 and Figure $5 A$ show that the preperfusion rates of REM-on neurons were greatly reduced by 8 -OH-DPAT perfusion with a median reduction of $89 \%$ for active REM discharge [ $p=0.004$, Wilcoxon signed rank test (WSRT)], whereas there were minimal or no effects on the discharge activity of Wake/ REM-on neurons (Figure $5 B$ ) in which the median discharge rate reduction was $1.5 \%$ (not significant, WSRT). In all cases, REM-on neurons decreased their discharge activity within 2 min of the calculated time that 8-OH-DPAT reached the probe adjacent to the recorded neuron.

Figure 6 graphs the mean percentage discharge rate changes across all behavioral states after 8-OH-DPAT in each member of the REM-on and Wake/REM-on populations. It can been seen that the distributions do not overlap; they are statistically different at the $p=<0.0001$ level (Mann-Whitney $U$ test). Note that the small increases and decreases in Wake/REM-on neurons are nearly equally distributed around zero, which is compatible with a null effect. Even the state-related discharge of the single Wake/
REM-on neuron with a moderate $(41 \%)$ 8-OH-DPAT-induced decrease in rate appears to be compatible with the hypothesis that $5-\mathrm{HT}_{1 \mathrm{~A}}$ inhibition in wakefulness leads to an REM-on discharge profile. Of all the Wake/REM-on units, this one had the highest active REM/AW discharge ratio (1.49) and was thus most like the REM-on neurons (defined by a ratio $>2$ ). These data are thus compatible with this neuron having a moderate but not strong inhibition in wakefulness. Finally, within the entire population of units, the active REM/AW discharge ratio for each unit (measuring the relative degree of suppression of discharge in wakefulness) correlated positively and strongly with the degree of suppression of the unit of discharge after 8-OH-DPAT (Spearman's $\rho=0.67 ; p<0.005)$.

\section{DISCUSSION}

This study has taken advantage of a novel methodology combining microdialysis and single-unit recording in the freely behaving animal to show that REM-on neurons in the cholinergic LDT and PPT nuclei are inhibited by the $5-\mathrm{HT}_{1 \mathrm{~A}}$ agonist $8-\mathrm{OH}-\mathrm{DPAT}$, whereas Wake/REM-on neurons are not. Because serotonergic neurons in the DRN have their highest discharge rate and concomitant serotonin release in wakefulness and have projections to LDT/PPT, these data provide strong support to the reciprocal interaction model (McCarley and Massaquoi, 1992). This model postulates that REM-on neurons are under monoaminergic inhibition in wakefulness but become active just before and during REM sleep as a result of monoaminergic disinhibition, because DRN serotonergic neurons virtually cease discharging during this part of the sleep cycle. That the experimental 8-OH-DPAT administration mimics natural serotonergic inhibition in wakefulness is further strengthened by the strong correlation between natural REM/wakefulness discharge ratios and the degree of suppression of REM discharge by $8-\mathrm{OH}-\mathrm{DPAT}$. Our study also suggests that serotonergic inhibition of REM-on neurons via a $5-\mathrm{HT}_{1 \mathrm{~A}}$ receptor is responsible for the lower discharge activity of these neurons during AW.

Although previous studies have recorded LDT and PPT neurons across behavioral state, the present study is the first to report behavioral state-related discharge rates of LDT- and PPTlocalized neurons in the freely moving animal. El Mansari et al. (1989) recorded the activity of neurons from the rostral pontine tegmentum in freely behaving cats, but the area of recording was not restricted to the PPT (although PPT was included). Moreover, in this study the recordings were biased toward those units antidromically identified as projecting to thalamus. Steriade et al. (1990) recorded single units from the LDT and PPT in headrestrained cats and hence could not describe activity related to movement in active wakefulness (AW). Kayama et al. (1992) recorded single units in sleep-deprived, head-restrained rats. Given the possibility that cells in brainstem areas can exhibit discharge patterns that are related to head and neck movements (Siegel et al., 1977), it thus becomes important that the present data support the findings of these earlier reports in headrestrained animals. For REM-on neurons, El Mansari et al. (1989) reported that the mean discharge rate was $\sim 3$ spikes/sec during AW, whereas in our study we found that the mean discharge rate for REM-on neurons during AW was 1.45 spikes/sec (Table 1). The remaining two studies did not look at firing rates during the AW state.

Identification of the recorded neurons as cholinergic is consistent, although tentative, with several criteria (see Materials and Methods). First, histological reconstruction of our recording 


\section{REM-on neuron}

Active wake

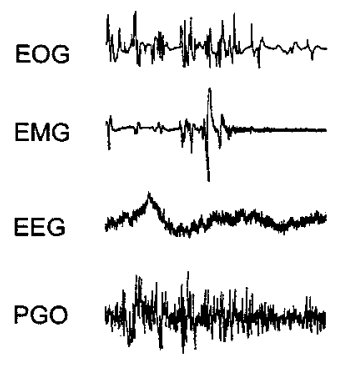

Unit

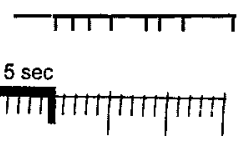

Figure 3. Digitized inkwriter record of REM-on unit discharge in the different behavioral states and during perfusion of 8-OH-DPAT. The first panel is active wakefulness, with activity in both EOG and EMG (indicating somatomotor activity). Note the low level of unit activity, which continues in non-REM sleep but increases more than twofold in REM sleep. 8-OHDPAT dramatically suppressed unit activity in the same REM period. Voltage gain for active-wake EMG is $200 \mu \mathrm{V}$.
Figure 4. Digitized inkwriter record of Wake/REM-on unit discharge in the different behavioral states and during perfusion of 8-OH-DPAT. The unit shows a high level of activity in both active wakefulness and REM sleep relative to non-REM sleep. Note that 8-OH-DPAT does not suppress unit discharge in REM sleep.
nonREM sleep

REM sleep

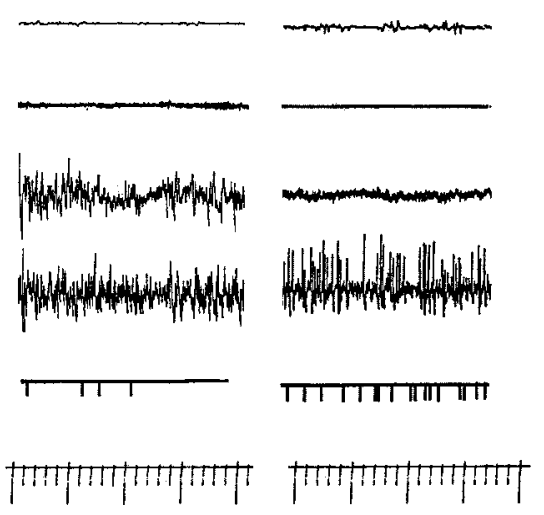

REM sleep after 8-OH-DPAT

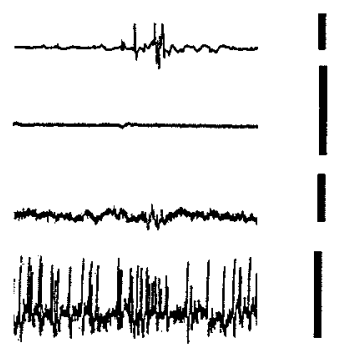

$100 \mathrm{uV}$

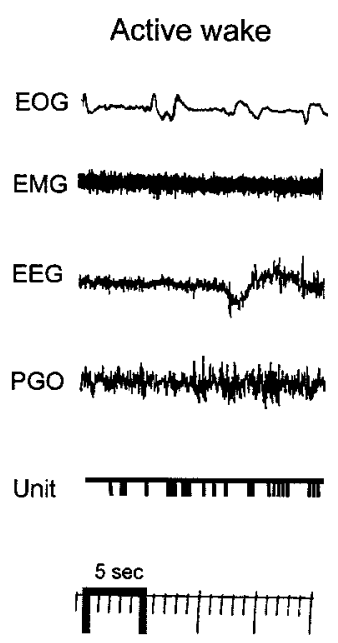

Wake/REM-on neuron

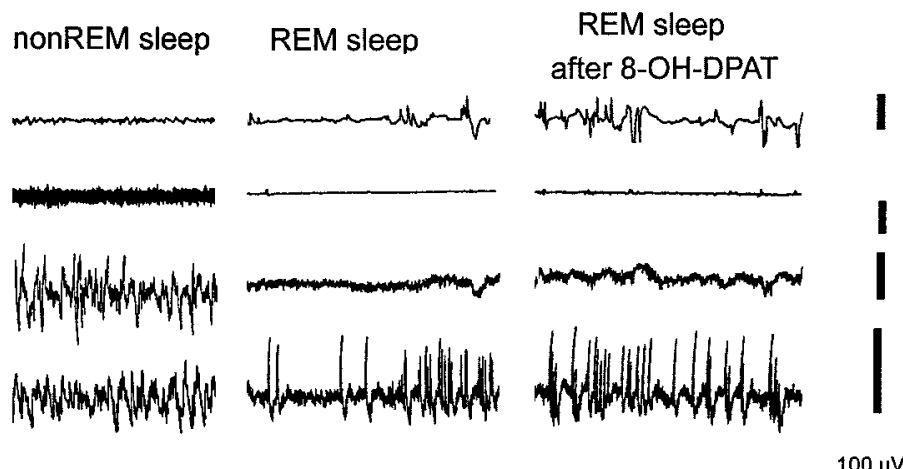

TI T

$100 \mathrm{uV}$

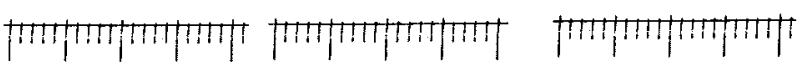

tracts indicated that all reported cells were recorded in the anatomically defined cholinergic zones of LDT or PPT. Anatomical studies indicate that in these regions $\sim 80 \%$ (Steriade et al., 1990; Jones, 1993) of the large neurons ( $>20 \mu \mathrm{m}$ cell body diameter) are ChAT-positive, indicative of their being cholinergic. Second, the recording method used fine wires of 32 and 64 $\mu \mathrm{m}$ diameter, which is a method that preferentially records larger cells $(>20 \mu \mathrm{m})$. Finally, studies have argued that cells in the cholinergic LDT/PPT with long-duration action potentials (Steriade et al., 1990) or slow conduction velocity (El Mansari et al., 1989) are likely to be cholinergic. The majority of the neurons recorded by us had long-duration action potentials. We thus believe that the large majority of the two types of cells that we recorded (REM-on and Wake/REM-on) are cholinergic.

The finding that only a subpopulation of the recorded LDT/ PPT cells were inhibited by 8-OH-DPAT is consistent with rat pontine slice data in which, using combined intracellular recording and labeling to confirm the cholinergic identity of the recorded cell, our laboratory found that $64 \%$ of the cholinergic neurons in the LDT/PPT were inhibited by serotonin (Luebke et al., 1992). However, it is not possible to obviously determine whether the cells recorded in vitro are REM-on or Wake/REMon, because there are no purely electrophysiological criteria sufficient to identify the state-related characteristics of the cell. The different percentages of LDT/PPT neurons that are inhibited by serotonin or serotonin agonists in vitro (64\%) compared with our in vivo findings $(36.4 \%)$ may be attributable to anatomical differences between species (rat vs cat) and/or different concentrations of agents at the receptors. Luebke et al. (1992) did not do a concentration-response study, and their bath-applied serotonin agonists may have had a higher concentration at receptor sites than in the present study. Further research is needed to determine whether differential serotonergic inhibition in LDT/PPT results from differing serotonergic innervation and/or different receptor distribution or sensitivity on different neurons. Anatomical studies in the cat of the percentage of mesopontine cholinergic neurons with $5-\mathrm{HT}_{1 \mathrm{~A}}$ receptors are needed, as has been done previously in rat forebrain (Kia et al., 1996).

Recently, Sakai and Koyama (1996), using microinotophoresis in head-restrained cats, looked at the effect of serotonin on REM-on neurons recorded primarily from the peri locus ceruleus $\alpha$ region, which is an area in the dorsal pontine tegmentum close 


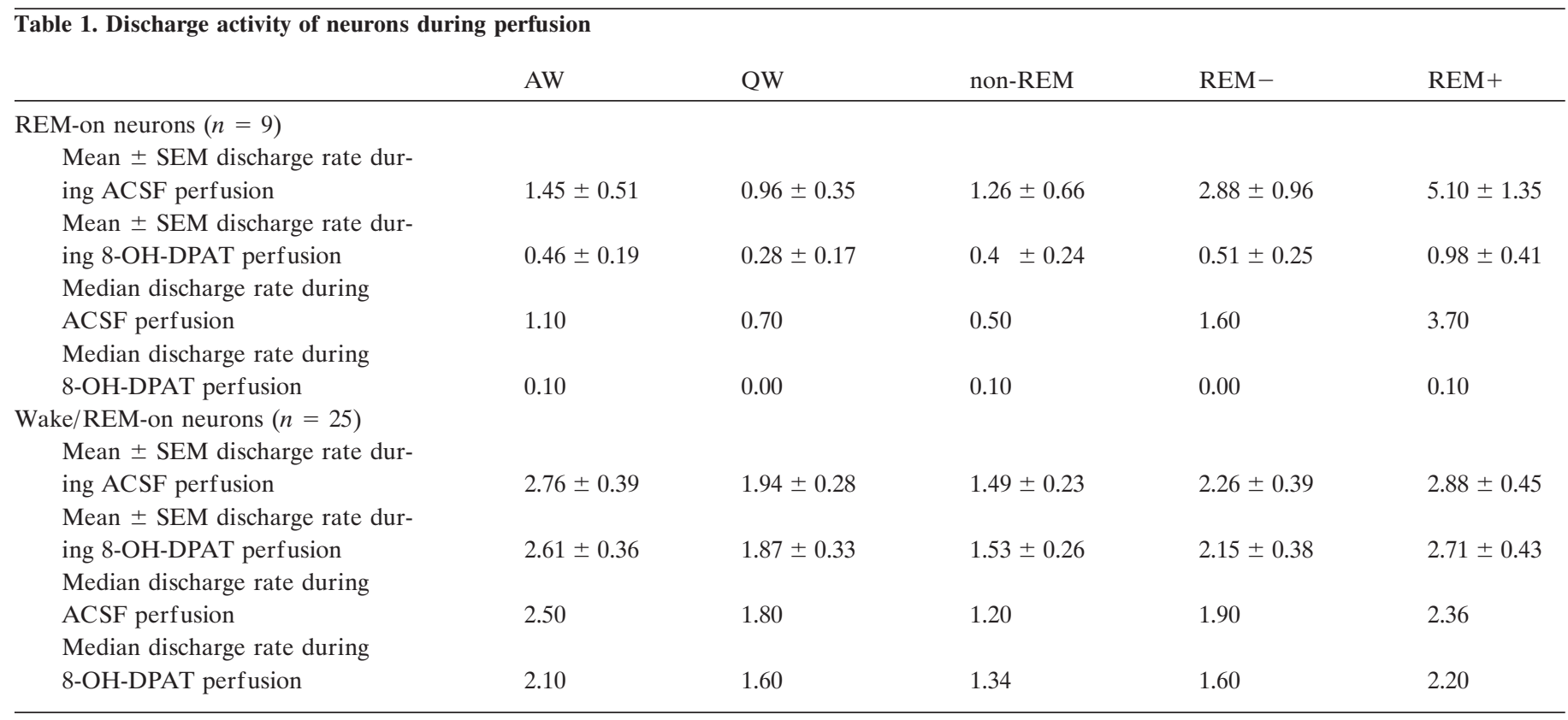

Discharge activity of neurons during ACSF and 8-OH-DPAT perfusion (in discharges per second). See Results for discussion.

to, but not identical with, the LDT/PPT zones recorded in the present study. Interestingly, they found that serotonin had no effect on the discharge activity of REM-on neurons in this region, indicating that the inhibitory action of serotonin and its agonists on REM-on cells may be regionally specific within the pontine tegmentum. Further work is needed to compare the characteristics of neurons in these two REM sleep-related areas and to fully determine the neurotransmitter inputs that determine the REM-on discharge pattern of cells in the two areas. The present in vivo results are entirely compatible with extensive in vitro studies indicating that serotonin in the LDT/PPT acts through a 5- $\mathrm{HT}_{1 \mathrm{~A}}$ receptor (Muhlethaler et al., 1990; Luebke et al., 1992; Leonard and Llinas, 1994), although an immunohistochemical study has reported the presence of $5-\mathrm{HT}_{2}$ receptors in the LDT/PPT (Morilak and Ciranello, 1993). Sanford et al. (1996) have suggested that $\mathrm{LDT} / \mathrm{PPT} 5-\mathrm{HT}_{1 \mathrm{~A}}$ receptors might be too few to mediate serotonin effects compared with DRN. This latter conclusion might be revised by our data indicating only a minority (one of three to one of five) of LDT/PPT neurons are inhibited by $5-\mathrm{HT}_{1 \mathrm{~A}}$ agonists and indeed by the more recent work of Horner et al. (1997).

The present paper has described a novel method for simultaneously recording the extracellular discharge activity of single neurons while exposing the recorded cell to drugs perfused via an adjacent microdialysis probe. This method has several strengths, including that it can be done in the intact, freely behaving animal. Using the microdialysis probe for perfusion of drugs in the vicinity of the recorded cell also allows fine control over the concentration and duration of the drug presentation. Existing alternatives to this method appear to have several disadvantages. They require restrained animals (microinotophoresis), do not control the drug concentration presented (microinotophoresis, unit recording combined with microinjections), and/or are unable to reliably maintain unit recordings during the drug administration (microinjections). The combination of microdialysis and unit recording also affords the opportunity of sampling the extracellular environment for analysis of neurotransmitter levels while the unit is recording. Further confirming the validity of the procedure, we observed in one preliminary experiment that perfusion of a $300 \mathrm{nM}$ concentration of the specific $5-\mathrm{HT}_{1 \mathrm{~A}}$ antagonist 4-iodo- $N$-[2-[4-(methoxyphenyl)-1-piperazinyl]ethyl]$N$-2-pyrinyl-benzamide increased the discharge rate of a REM-on neuron during wakefulness. These preliminary data further underscore the utility of this neuropharmacological-neuro physiological methodology for exploring behavioral state control.

\section{Implications for behavioral state control}

Finally, in terms of a model of the brainstem regulation of REM sleep, the present results are consistent with the following sequence of neural events. During wakefulness, the discharge rate of DRN serotonergic neurons (and noradrenergic neurons of the locus ceruleus) is maximal, and within the mesopontine cholinergic nuclei, the higher levels of serotonin release associated with waking inhibit the REM-on cells but do not affect the Wake/ REM-on cells. The high discharge rate of the Wake/REM-on cells during waking is thought to contribute to the activated (desynchronized) cortical EEG typical of wakefulness via their projections to the thalamus (El Mansari et al., 1989; Steriade et al., 1990). As the animal becomes drowsy and begins to sleep, the discharge rate of serotonergic (and adrenergic) neurons slows progressively until the neurons are virtually silent in REM sleep, resulting in a decrease in serotonin release from serotonin synapses in terminal areas such as the LDT/PPT (Strecker et al., 1998). This decrease in monoamine release in the LDT/PPT results in a disinhibition of the REM-on cholinergic neurons, and they begin to discharge. During REM sleep, both populations of LDT/PPT cholinergic neurons discharge, and we speculate that the REM-on subpopulation is primarily responsible for the descending input to lower brainstem areas that generate many of the physical signs of REM sleep. The REM-on neurons also project to the thalamus, wherein they may functionally join with the Wake/REM-on neuronal projections to promote the activated EEG characteristic of REM sleep (El Mansari et al., 1989, Steriade et al., 1990). (Parenthetically, we note that although the data are not yet as strong, we believe that the effects of the companion 
A

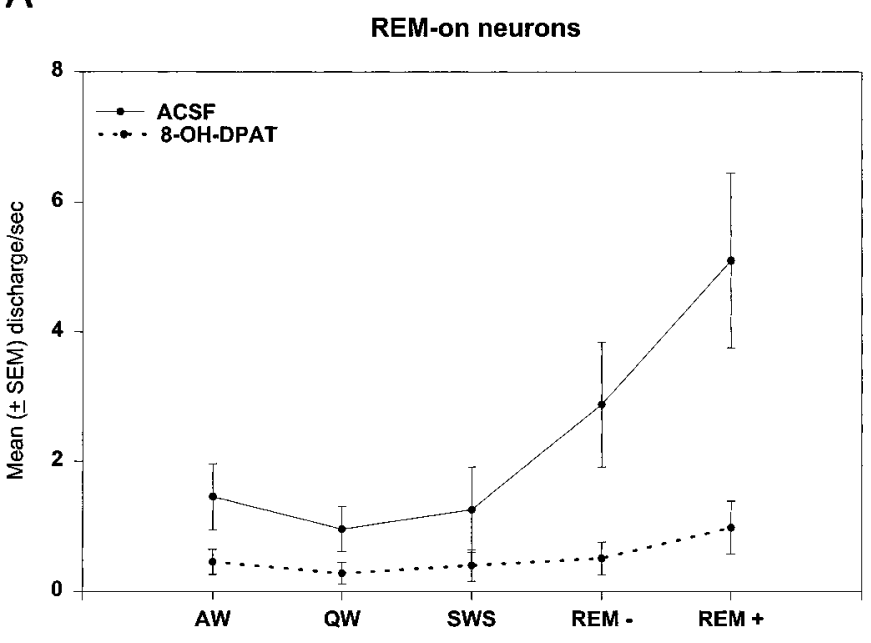

B

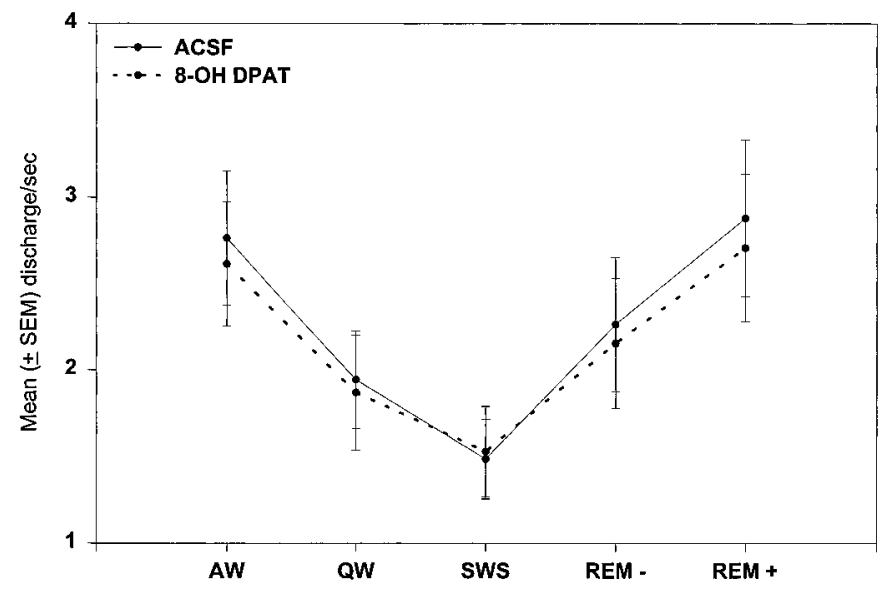

Figure 5. $A$, REM-on units $(n=9)$. Grand mean \pm SEM of discharge rate in each behavioral state before (solid line, ACSF) and after (dotted line) $10 \mu \mathrm{M} 8-\mathrm{OH}-\mathrm{DPAT}$ was added to the perfusate. Note 8-OH-DPAT suppression of activity (highly statistically significant; see text and Table $1)$. $B$, Wake/REM-on units $(n=25)$. Grand mean \pm SEM of discharge rate before (solid line, ACSF) and after (dotted line) $10 \mu \mathrm{M} 8$-OH-DPAT was added to the perfusate. Note minimal effect of 8-OH-DPAT (not statistically significant; see text and Table 1). SWS, non-REM sleep.

monoamine noradrenaline parallel those of serotonin and have so indicated in this description.)

However, the neural mechanisms that initiate and terminate the REM sleep cycle are not as yet clearly understood. For example, it remains to be determined what neural mechanisms produce the slowing of monoaminergic neuronal discharge and thus may disinhibit LDT/PPT neurons, although current work offers some clues. It appears likely that multiple mechanisms modulate the activity of DRN serotonergic neurons. These neurons are inhibited by serotonin agonists that act at the 5-HT autoreceptor to produce a decrease in discharge (Thakkar et al., 1998), a decrease in serotonin release, and a threefold increase in REM sleep (Portas et al., 1996). Evidence also supports a role for GABA and adenosine in the regulation of serotonergic activity (Nitz and Siegel, 1997; Porkka-Heiskanen et al., 1997). A repeated finding in all of these studies is that increases in serotonergic activity produce an increase in waking, whereas manipulations decreasing serotonergic activity increase the amount of REM sleep.

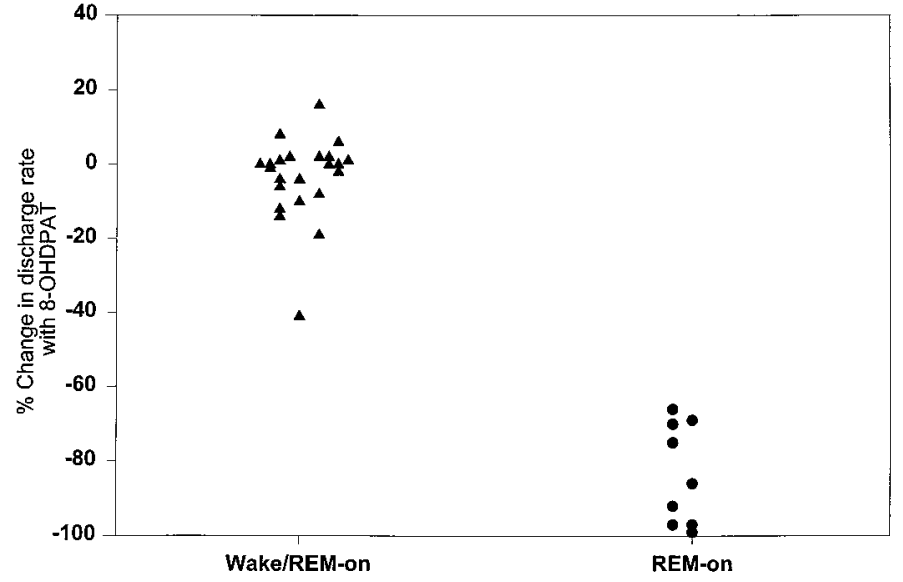

Figure 6. Scattergram showing the mean percentage change (across all behavioral states) in the discharge rate during 8-OH-DPAT perfusion for each Wake/REM-on unit and each REM-on unit. Note that the distributions do not overlap and that most of the Wake/REM-on unit values are clustered around zero. See Results for further discussion.

It is similarly likely that a variety of neurotransmitter inputs modulate the activity of LDT/PPT cholinergic neurons. One neurotransmitter input that may be particularly relevant to behavioral state control is that of noradrenaline. We predict that noradrenaline acting through $\alpha_{2}$ receptors will be found to have the same differential inhibitory effects in vivo as serotonin: inhibiting REM-on but not Wake/REM-on LDT/PPT neurons. This prediction readily leads itself to testing through the experimental method introduced here. Other populations of neurons may show also differential neuromodulation relevant to particular behaviors and responses. For example, anatomical data indicate subpopulations of cholinergic neurons with and without $5-\mathrm{HT}_{1 \mathrm{~A}}$ receptors in the medial septum and diagonal band of Broca (Kia et al., 1996). Using simultaneous unit recording and microdialysis technique in the freely behaving animal may allow specification of particular behavior(s) associated with the subpopulations.

\section{REFERENCES}

Amatruda III TT, Black DA, McKenna TM, McCarley RW, Hobson JA (1975) Sleep cycle control and cholinergic mechanisms: differential effects of carbachol injections at pontine brain stem sites. Brain Res 98:501-515.

Berman AL (1968) The brainstem of the cat. Madison, WI: The University of Wisconsin.

El Mansari M, Sakai K, Jouvet M (1989) Unitary characteristic of presumptive cholinergic tegmental neurons during the sleep-waking cycle in freely moving cats. Exp Brain Res 76:519-529.

Foote SL, Bloom FE, Aston-Jones G (1983) Nucleus locus coeruleus: new evidence of anatomical and physiological specificity. Physiol Rev 252:117-127.

George R, Haslett WL, Jenden DJ (1964) A cholinergic mechanism in the pontine reticular formation: induction of paradoxical sleep. J Neuropharmacol 3:541-552.

Greene RW, Gerber U, McCarley RW (1989) Cholinergic activation of medial pontine reticular formation neurons in vitro. Brain Res 476:154-159.

Hobson JA, McCarley RW, Wyzinski PW (1975) Sleep cycle oscillation: reciprocal discharge by two brainstem neuronal groups. Science 189:55-58.

Honda T, Semba K (1994) Serotonergic synaptic input to cholinergic neurons in the rat mesopontine tegmentum. Brain Res 647:299-306.

Horner RL, Sanford LD, Annis D, Pack AI, Morrison AR (1997) Serotonin at the laterodorsal tegmental nucleus suppresses rapid eye movement sleep in freely behaving rats. J Neurosci 17:7541-7552. 
Ito K, McCarley RW (1984) Alterations in membrane potential and excitability of cat medial pontine reticular formation neurons during changes in naturally occurring sleep-wake states. Brain Res 292:169-175.

Jacobs BL, Fornal CA (1991) Activity of brain serotonergic neurons in the behaving animal. Pharmacol Rev 43:563-578.

Jones BE (1993) The organization of central cholinergic systems and their functional importance in sleep-waking states. Prog Brain Res 98:61-71.

Jouvet M (1962) Recherches sur les structures nerveuses et les mechanismes responsales des differentes du sommeil physiologique. Arch Ital Biol 100:125-206.

Kayama Y, Ohta M, Jodo E (1992) Firing of "possibly" cholinergic neurons in the rat laterodorsal tegmental nucleus during sleep and wakefulness. Brain Res 569:210-220.

Kia HK, Brisorgueil MJ, Daval G, Langlois X, Hamon M, Verge D (1996) Serotonin1A receptors are expressed by a subpopulation of cholinergic neurons in the rat medial septum and diagonal band of Broca- a double immunocytochemical study. Neuroscience 74:143-154.

Kodama T, Lai YY, Siegel JM (1992) Enhancement of acetylcholine release during REM sleep in the caudomedial medulla as measured by in vivo microdialysis. Brain Res 580:348-350.

Leonard CS, Llinas R (1994) Serotonergic and cholinergic inhibition of mesopontine cholinergic neurons controlling REM sleep: an in vitro electrophysiological study. Neuroscience 59:309-330.

Leonard TO, Lydic R (1997) Pontine nitric oxide modulates acetylcholine release, rapid eye movement sleep generation, and respiratory rate. J Neurosci 17:774-785.

Luebke JI, Greene RW, Semba K, Kamondi A, McCarley RW, Reiner PB (1992) Serotonin hyperpolarizes cholinergic low-threshold burst neurons in the rat laterodorsal tegmental nucleus in vitro. Proc Natl Acad Sci USA 89:743-747.

Lydic R, McCarley RW, Hobson JA (1987) Serotonin neurons and sleep. I. Long term recordings of dorsal raphe discharge frequency and PGO waves. Arch Ital Biol 125:317-343.

McCarley RW, Hobson JA (1975) Neuronal excitability modulation over the sleep cycle: a structural and mathematical model. Science 189:58-60.

McCarley RW, Massaquoi SG (1992) Neurobiological structure of the revised limit cycle reciprocal interaction model of REM sleep cycle control. J Sleep Res 1:132-137.

McCarley RW, Greene RW, Rainnie DG, Portas CM (1995) Brainstem neuromodulation and REM sleep. Semin Neurosci 7:341-354.

McGinty DJ, Harper RM (1976) Dorsal raphe neurons: depression of firing during sleep in cats. Brain Res 101:569-575.

Mitani A, Ito K, Hallanger AE, Wainer BH, Kataoka K, McCarley RW (1988) Cholinergic projections from the laterodorsal and pedunculopontine tegmental nuclei to the pontine gigantocellular tegmental field in the cat. Brain Res 451:397-402.

Morilak DA, Ciaranello RD (1993) 5-HT2 receptor immunoreactivity on cholinergic neurons of the pontomesencephalic tegmentum shown by double immunofluorescence. Brain Res 627:49-54.

Muhlethaler M, Khateb A, Serafin M (1990) Effects of monoamines and opiates on pedunculopontine neurones. In: The diencephalon and sleep (Mancia M, Marini G, eds), pp 367-378. New York: Raven.

Nitz D, Siegel J (1997) GABA release in the dorsal raphe nucleus: role in the control of REM sleep. Am J Physiol 273:R451-R455.

Porkka-Heiskanen T, Strecker RE, Stenberg D, Bjorkum AA, McCarley RW (1997) GABA and adenosine inhibit the dorsal raphe nucleus and increase REM sleep as studied by microdialysis [abstract]. Sleep Res 26:35.
Portas CM, McCarley RW (1994) Behavioral state-related changes of extracellular serotonin concentration in the dorsal raphe nucleus: a microdialysis study in the freely moving cat. Brain Res 648:306-312.

Portas CM, Thakkar M, Rainnie D, McCarley RW (1996) Microdialysis perfusion of 8-hydroxy-2-(di- $n$-propylamino)tetralin (8-OH-DPAT) in the dorsal raphe nucleus decreases serotonin release and increases rapid eye movement sleep in the freely moving cat. J Neurosci $16: 2820-2828$.

Sakai K, Koyama Y (1996) Are there cholinergic and non-cholinergic paradoxical sleep-on neurones in the pons? NeuroReport 7:2449-2453.

Sanford LD, Ross RJ, Seggos AE, Morrison AR, Ball WA, Mann GL (1994) Central administration of two 5-HT receptor agonists: effect on REM sleep initiation and PGO waves. Pharmacol Biochem Behav 49:93-100.

Sanford LD, Tejani-Butt SM, Ross RJ, Morrison AR (1996) Elicited PGO waves in rats: lack of 5-HT1A inhibition in putative pontine generator region. Pharmacol Biochem Behav 53:323-327.

Semba K, Fibiger HC (1992) Afferent connections of the laterodorsal and the pedunculopontine tegmental nuclei in the rat: a retro- and antero-grade transport and immunohistochemical study. J Comp Neurol 323:387-410.

Siegel JM, McGinty DJ, Breedlove SM (1977) Sleep and waking activity of pontine gigantocellular field neurons. Exp Neurol 56:553-573.

Steininger TL, Wainer BH, Blakely RD, Rye DB (1997) Serotonergic dorsal raphe nucleus projections to the cholinergic and noncholinergic neurons of the pedunculopontine tegmental region: a light and electron microscopic anterograde tracing and immunohistochemical study. J Comp Neurol 382:302-322.

Steriade M, Datta S, Pare D, Oakson G, Curro DR (1990) Neuronal activities in brain-stem cholinergic nuclei related to tonic activation processes in thalamocortical systems. J Neurosci 10:2541-2559.

Strecker RE, Porkka-Heiskanen T, Thakkar MM, Dauphin L, McCarley RW (1998) Neuromodulation of the pedunculopontine tegmental nucleus (PPT) in the control of REM sleep as studied by microdialysis. Sleep [Suppl] 21:22.

Thakkar M, Mallick BN (1991) Effect of REM sleep deprivation on rat brain acetylcholinesterase. Pharmacol Biochem Behav 39:211-214.

Thakkar M, Inglis W, Rainnie D, Green R, McCarley RW, Semba K (1995) Effect of discrete ibotenic acid lesions of the mesopontine tegmentum on sleep and waking states in the rat. Soc Neurosci Abstr 2:957.

Thakkar M, Portas C, McCarley RW (1996) Chronic low-amplitude electrical stimulation of the laterodorsal tegmental nucleus of freely moving cats increases REM sleep. Brain Res 723:223-227.

Thakkar M, Strecker RE, McCarley RW (1997) The 5-HT 1 agonist 8-OH-DPAT inhibits REM-on neurons but has no effect on waking and REM-on neurons: a combined microdialysis and unit recording study [Abstract]. Sleep Res 26:52.

Thakkar M, Strecker RE, McCarley RW (1998) Perfusion of a 5-HT $1 \mathrm{~A}$ agonist in the dorsal raphe inhibits REM-off neurons: a combined unit recording and microdialysis study. Sleep [Suppl] 21:3.

Ursin R, Sterman MB (1981) A manual for standardized scoring of sleep and waking states in the adult cat, Los Angles: Brain Information Service, Brain Research Institute, University of California. pp 1-103.

Webster HH, Jones BE (1988) Neurotoxic lesions of the dorsolateral pontomesencephalic tegmentum-cholinergic cell area in the cat. II. Effects upon sleep-waking states. Brain Res 458:285-302.

Williams JA, Reiner PB (1993) Noradrenaline hyperpolarizes identified rat mesopontine cholinergic neurons in vitro. J Neurosci 13: 3878-3883. 